Instituto Internacional de Investigación y Desarrollo Tecnológico Educativo INDTEC, C.A.

DOI: https://doi.org/10.29394/Scientific.issn.2542-2987.2018.3.9.19.348-361

OAI-PMH: http://www.indteca.com/ojs/index.php/Revista Scientific/oai

Ensayo Original / Original Essay

\title{
La Praxis de Enfermería: Una Vocación con Sentido Axiológico y Humanista
}

Autor: Rubén Eliut Hernández Ortega Universidad Latinoamericana y del Caribe, ULAC rubenho1977@gmail.com

Caracas, Venezuela

\section{Resumen}

El presente ensayo tiene como propósito abordar la praxis de enfermería desde una visión axiológica y humanista, basado en la filosofía del cuidado humanizado descrito por Watson Jean, en tertulia dialógica con Katherine Núñez (2017), donde comprende al cuidado desde lo existencial fenomenológico y espiritual; incluyendo para ello una acción humana moral y ética, propia de la profesión de enfermería, alcanzando además una relación terapéutica primordial entre humanos, basada en el respeto y la intersubjetividad. Y la teoría del desarrollo moral de Lawrence Kohlberg (1997), considerando que la madurez psicológica y acción moral no necesariamente van paralela a la maduración biológica del ser humano, por lo que la conciencia moral sigue un proceso de crecimiento o de madurez a lo largo de la vida de las personas. En base a ello, la praxis profesional debe ir enmarcada con las exigencias y las transformaciones de los sistemas de salud del mundo, por tanto, la formación, la capacitación continua, la reflexividad e interreflexividad sobre el accionar de su practicidad debe ser sistemático, continuo y profundo, consciente de que su actuación e interacción en un ámbito sociocultural cambiante es determinante para ofrecer ayuda terapéutica, además de restaurar la salud y proteger la vida.

Palabras clave: aptitud; humanismo; personal paramédico. 


\title{
The Practice of Nursing: A Vocation with Axiological and Humanist Sense
}

\begin{abstract}
The purpose of this essay is to approach nursing praxis from an axiological and humanistic perspective, based on the philosophy of humanized care described by Watson Jean, in a dialogical discussion with Katherine Núñez (2017), where he understands the phenomenological and spiritual existential care; including for this a moral and ethical human action, typical of the nursing profession, also reaching a primordial therapeutic relationship between humans, based on respect and intersubjectivity. And the theory of moral development of Lawrence Kohlberg (1997), considering that psychological maturity and moral action do not necessarily parallel the biological maturation of the human being, so that the moral conscience follows a process of growth or maturity throughout of people's lives. Based on this, professional praxis should be framed with the demands and transformations of the world's health systems, therefore, training, continuous training, reflexivity and interreflexivity on the action of its practicality should be systematic, continuous and deep, aware that their action and interaction in a changing sociocultural environment is crucial to offer therapeutic help, in addition to restoring health and protecting life.
\end{abstract}

Keywords: aptitude; humanism; paramedical personnel.

Date Received: 10-01-2018

Date Acceptance: 05-03-2018 


\section{Introducción}

A partir de los preceptos de la educación se hace indispensable asumir los soportes que rigen la misma a nivel mundial y que además abarcan los principios y valores descritos en la Constitución de la República Bolivariana de Venezuela (1999), y la Ley Orgánica de Educación (2009).

Sobre la base de lo anteriormente señalado se crean las consideraciones respectivas para abordar el tema de la praxis de la enfermería ligada a la educación, siendo que dicha ciencia parte de unos principios filosóficos presentes en la concepción curricular y que además incluyen una relación estrecha entre la vocación, las competencias, los valores humanos y la practicidad humanística.

Por lo tanto, el presente ensayo tiene como propósito abordar la praxis de la enfermería desde una visión axiológica con sentido humanista, sustentado el mismo en la teoría del cuidado humano de Jean Watson, citado por (Guillaumet, García, Casacuberta y Serra, 2005, pág. 128), donde sostiene que el cuidado de enfermería se centra en enfoques filosóficos (existencialfenomenológico) desde lo espiritual; además concibe el cuidado como una acción moral y ética característica de la profesión de la enfermería, es decir; considera el cuidado humano como una relación terapéutica primordial entre seres humanos, visualizándolo además como relacional, transpersonal e intersubjetiva.

En ese mismo sentido, resulta importante analizar, comprender y explicar la relación causa-efecto del comportamiento humano, de acuerdo con los aspectos señalados por Kohlberg (1997a), en la teoría del desarrollo moral; citado por (Reluz y Cajachahua, 2011a, pág. 103), donde hace énfasis al proceso de crecimiento y madurez de la conciencia moral, la reflexión y el razonamiento que determina la manera de actuar de las personas.

De acuerdo con lo anteriormente señalado, el desarrollo moral de los profesionales de Enfermería se encuentra básicamente en el conocimiento de 
tipo social con relación a la conjugación praxis-sociedad y que debe ser reforzado constantemente con principios axiológicos y fundamentados en la reflexividad de la acción ante la interacción ambiente, sociedad y praxis.

Significa entonces, que el profesional de enfermería tiene las herramientas para realizar una praxis acorde con las exigencias $y$ transformaciones de los sistemas de salud del mundo, por tanto, la formación, capacitación continua, la reflexividad e interreflexividad sobre la acción que cada uno realiza, sin duda ayudará a internalizar, mejorar y profundizar la actuación e interacción de su practicidad en un ámbito sociocultural cambiante.

En base a las consideraciones anteriores, a medida que los profesionales de enfermería desempeñen sus funciones en forma óptima, la sociedad se beneficiará de una atención basada en modelos de calidad y excelencia propios de las políticas de estado consagradas en los textos jurídicos de la República Bolivariana de Venezuela, en cuanto a la promoción de salud y la recuperación de ésta, por medio de los cuidados humanos fundamentados en competencias y en valores.

En ese mismo sentido se habla de la significación de las habilidades profesionales desde el saber hacer y el saber convivir, donde se debe tener en cuenta que ellas se convierten en el objeto de la profesión y del cuidado de enfermería al individuo sano o enfermo en su relación reciproca con el medio social en el cual se desenvuelve, tomando en cuenta las necesidades humanas como base fundamental en esa interacción entre la praxis, la sociedad y el ambiente.

\section{Desarrollo}

\subsection{La vocación como esencia de la praxis de enfermería.}

De acuerdo con Agrazal (2016), la esencia de ser enfermera(o) atrae a una "fuerza inspiradora llamada vocación", donde no solo se tiene un deseo efusivo para el trabajo y el logro del bienestar de las demás personas, sino que 
está enmarcado en el beneficio que le otorga la praxis de la enfermería en el cuidado de la vida del ser humano. La autora refiere además que el desarrollo de la vocación de la enfermería se construye por medio de los factores, condiciones y elementos que emergen desde el interior de cada persona que elige ser enfermera(o), y se concatena con el desarrollo de las habilidades y competencias para promover salud, prevenir enfermedades y brindar cuidados humanos a la persona, familia y comunidad.

De modo que, cuando la vocación de ser enfermera(o), es parte de la vida, se pasa a realizar la praxis con una óptima responsabilidad social, brindando atención con calidad humana, donde más allá de la exigencia del sistema de salud, esto conlleva a reconocer el qué, el por qué y para qué de la profesión.

Debido a esto, es importante destacar que la vocación centra sus propósitos en esa voz interior que conlleva a la persona a realizar acciones que se entrelazan con el ser ontológico de la profesión, y que se complementan con la formación y capacitación profesional.

En términos concisos, la vocación de enfermería está ligada a la praxis que diariamente realizan éstos profesiones y que conllevan a una internalización del proceder y el accionar fundamental como razón de ser del cuidado humano.

Por esta razón la vocación de enfermería pasa a ser un precepto individual, arraigado en la concepción del ser humano y complementado con los más altos estándares de formación, para asumir y ejercer una praxis cónsona con lo establecido en el ejercicio profesional.

Resulta oportuno destacar que la formación del profesional de enfermería lleva consigo una serie de elementos axiológicos, teóricos y humanistas que construyen los saberes enfermeros y permiten la reflexión crítica constante del cuidado brindado, aflorando así la vocación como factor indispensable en la esencia de esa acción humana. 
Con relación a ello, un estudio cualitativo realizado por (Camejo, 2017, pág. 242), destaca que "como hallazgo principal se conoció que la mayoría de los estudiantes de enfermería no conocen el futuro de la carrera".

Sin duda, partiendo de los preceptos de este autor, es evidente que la vocación en enfermería es un fundamento humano que se inicia en la formación y que además lleva consigo una concepción real de lo que el futuro profesional asumirá como principio para basar su praxis en el contexto social.

\subsection{La Praxis de enfermería desde la concepción humanista.}

Basándome en la definición utilizada por Paulo Freire en la pedagogía de oprimido (1979), la praxis es una "reflexión y acción de los hombres sobre el mundo para transformarlo". Citado por (Masi, 2008, pág. 78).

En relación con este concepto, es importante asumir la praxis de enfermería desde una visión humana, cargada de esencia moral, ética y axiológica fundamental, que conlleven a una reflexividad continua sobre la actuación profesional y de esta manera lograr la transformación social por medio del cuidado humano.

De manera que, la praxis conjuga la capacidad para comprender, reflexionar y actuar sobre los efectos estructurales y tradicionales que la sociedad concibe sobre los cuidados enfermeros, teniendo como base fundamental la interacción entre el saber, el hacer, el convivir y el ser; guiando así la acción y la disposición a actuar moral y correctamente.

En relación al termino "praxis de enfermería", (López y Torres, 2009a, pág. 37), señalan que el profesional de enfermería fundamenta su acción en analizar, comprender e interpretar las actividades propias de la disciplina, en cualquiera de las funciones que realice (asistencial, docente, administrativa e investigativa); por medio del conocimiento científico, las técnicas, las habilidades y los fundamentos axiológicos, desde una visión humanista; es decir, la praxis debe tener consigo la disposición de aprehender por medio de 
la reflexividad y el descubrir la esencia del arte de cuidar desde lo humano.

Por consiguiente, es importante que el profesional de enfermería comprenda, aprehenda e internalice que dicha concepción va intrínsecamente relacionada a su accionar humano frente a la sociedad tal como lo señalan (López y Torres, 2009b):

La praxis debe ser aprehendida como una función de la filosofía y esta no parte de categorías, no parte de conceptos generales y comprensivos, sino de los hombres; analiza lo que hace, pregunta por qué lo hace, enseña a saber lo que hace. (pág. 38).

En base a lo antes descrito, se puede señalar que la praxis de enfermería fundamenta sus principios en la axiología y el humanismo, por ello es importante incorporar aspectos fundamentales que direccionan la actuación de enfermería en la teoría del cuidado humano de Jean Watson, donde sostiene la existencia de un riesgo de deshumanización en el cuidado del paciente, debido a la restructuración de los sistemas de cuidado de salud a nivel mundial; para lo cual ésta autora hace énfasis en el rescate del aspecto humano, espiritual y transpersonal, aplicable en todas las funciones (asistenciales, administrativas, educativas y de investigación) de enfermería. Citado por (Guerrero, Meneses, De La Cruz, 2015a, pág. 131).

A partir de este precepto, se concibe que el significado ontológico de la enfermería es sin duda otorgado desde la formación, estructurado en las competencias del saber, del saber hacer, del convivir y del ser, los modelos filosóficos del cuidado humano, el código deontológico y la ley del ejercicio; sin embargo la falta de gestión del conocimiento, el capitalismo y la comercialización de las pólizas de salud, han causado una automatización en la forma de brindar los cuidados humanos, además de la deshumanización y la poco practicidad de valores sociales en el ejercicio del campo de la enfermería. 
Resulta oportuno considerar que las bases del cuidado de la enfermería crean sus cimientos en comprender los procesos de salud y los de enfermedad, por medio de la experiencia humana. Por tanto, la filosofía de la ciencia del cuidado es la definición de los resultados en la actividad netamente científica, relacionada con los aspectos humanísticos de la vida, además de la interrelación de la calidad de vida, incluida la muerte y la postergación de la vida.

En este mismo orden de ideas se pude considerar que el cuidado humano basado en valores, debe ser el pilar fundamental en todos los sistemas de salud del mundo actual, por tanto, se requiere de la formación y capacitación de profesionales que formen y conformen un sistema de salud humanístico, donde se esmeren en brindar cuidados altruistas, cultivando la sensibilidad para quien realiza los cuidados y para quien los recibe, con el propósito de generar cambios en la praxis de enfermería.

A manera de resumen, se puede decir que la praxis de enfermería incluye epistemológicamente una conexión entre la forma de realizar la práctica, incluyendo la acción humana y la axiología como fundamento esencial en el acto del cuidado humano y humanizado, en pro de lograr una actividad reflexiva, científicamente desarrollada e independiente.

\subsection{La praxis de enfermería y la axiología como eje fundamental.}

De acuerdo a los supuestos y valores descritos en la teoría del cuidado humano de Jean Watson, es evidente entonces que la vocación del profesional de la enfermería es una concepción interna que conlleva al sujeto a cumplir con los principios axiológicos, guiados por la dimensión espiritual de la vida, la capacidad para el crecimiento y el cambio, el valor y el afecto por la persona cuidada y la vida humana, la libertad de tomar decisiones ajustadas a la clínica y a la ciencia, además de la importancia de una relación interpersonal e intersubjetiva basada en el respeto y el amor al prójimo. 
Después de las consideraciones anteriores, es importante destacar que la praxis de enfermería tiene otro eje en el cual basa sus principios esenciales como es la axiología, cuyos enlaces se fortalecen con la adhesión de normas, principios y la propia esencia moral descrita en el código deontológico y la ley del ejercicio profesional de la enfermería.

Cabe agregar que la praxis de la enfermería hace énfasis en brindar un cuidado humano y humanizado, valorando por encima de todo a la vida y a la persona desde su integralidad; pero a su vez reflexionando y concientizando la acción humana que conlleva a la realización de esa praxis; donde el profesional debe incorporar elementos que favorezcan el cuidado desde lo técnico, lo científico y lo humanístico; conjugando plenamente las competencias del conocer, el hacer, el convivir y el ser.

En ese mismo sentido, tal como se ha venido señalando, la significación de las habilidades profesionales comprenden aspectos vocacionales, axiológicos y humanísticos planteados metodológicamente en el currículo desde el saber hacer y el saber convivir, no obstante, la importancia de asumir, interpretar y aplicar esa practicidad es sin duda el gran reto que tienen los profesionales de enfermería, ya que la integración de dichas competencias conforman el objeto de la profesión y del cuidado humano de enfermería, en pro de la humanización del mismo, tal como es señalado por Watson, "el ideal moral de enfermería es la protección, mejora y preservación de la dignidad humana. El cuidado humano involucra valores, voluntad y un compromiso para cuidar, conocimiento, acciones de cuidado y consecuencias". Citado por (Guerrero, Meneses, De La Cruz, 2015b, pág. 133).

De la misma manera, cabe hacer mención a la teoría del desarrollo moral de Kohlberg (1997b), en la cual considera que "la madurez psicológica y moral no necesariamente va paralela con la maduración biológica del ser humano". Por lo que "la conciencia moral sigue un proceso de crecimiento o de madurez", citado por (Reluz y Cajachahua, 2011b, pág. 103). 
En base a este autor, se puede recalcar que la conciencia moral se denota como un proceso de aprendizaje inalterable, en la que se adquieren nuevas estructuras de conocimiento, por lo que no se considera razonable que una vez aprendidas y puestas en funcionamiento por el sujeto dejen de actuar sustancialmente. Es decir; que de acuerdo con las etapas del desarrollo moral propuestas por este teórico, se puede evidenciar un campo de acción, valoración y conocimiento que está en la estructura mental del profesional de Enfermería, sin embargo, es importante precisar que pudiesen existir considerables fenómenos de desajuste en algunos que pudiesen incidir en una manera distinta de actuar frente a los problemas de causa laboral.

En términos concretos, desde los preceptos educativos se apuesta a la actuación de profesionales de enfermería que asuman sustancialmente su rol de cuidadores, acorde con las exigencias de formación y capacitación, capaces de gestionar cuidados humanos para transformar a la sociedad y al hombre en su esencia humana, internalicen dentro de su concepción filosófica la actuación moral, como elemento fundamental en la aplicación de su praxis diaria, basada en la vocación de servicio y los fundamentos axiológicos y humanistas.

Por lo que se considera que la praxis de enfermería comprende una actuación absoluta del sentido vocacional, una impregnación íntegra de valores sociales y un alto sentido humanista.

\section{Conclusiones}

La concepción filosófica de la praxis de la enfermería está enmarcada en un sustento jurídico establecido en las leyes de la República Bolivariana de Venezuela, sin embargo, es importante que el profesional de enfermería comprenda, aprehenda e internalice que dicha concepción va intrínsecamente relacionada a su accionar humano frente a la sociedad.

De tal manera que, esta acción humana debe ayudar a perfeccionar, 
internalizar y comprender desde lo espiritual, la razón de ser de la enfermería, que conlleva a la reflexividad de la acción realizada, en base a la caracterización del elemento primordial de la praxis; la vocación.

Por esta razón, los profesionales de enfermería tienen el reto y la capacidad fundamental de reestructurar administrativamente los sistemas de salud, por medio de la aplicación de principios praxiológicos y bajo el enfoque del cuidado humanizado.

Sin embargo, es esencial que los profesionales de enfermería puedan realizar su praxis bajo modelos de cuidado que engloben la teoría, la práctica, las habilidades, las destrezas, la investigación constante y continua en cada área de desempeño, la reflexión continua sobre los planes de cuidado, los métodos y las formas de cuidar, las técnicas que incorporen el manejo del conocimiento real de los valores de la profesión y los del sujeto cuidado, además del desarrollo de competencias del convivir que interconecten el por qué cuidar y el para qué cuidar; en base a un cuidado humanizado. Es decir; la praxis de enfermería debe esforzarse en humanizar los cuidados sustancialmente.

De acuerdo a lo antes planteado, es evidente que el profesional de enfermería posee una serie de herramientas que desde su formación se conjugan para realizar una praxis acorde con las exigencias y las transformaciones de los sistemas de salud del mundo, por tanto, la formación, la capacitación continua, la reflexividad e interreflexividad sobre el accionar de su practicidad debe ser sistemático, continuo y profundo, consciente de que su actuación e interacción en un ámbito sociocultural cambiante es determinante para ofrecer ayuda terapéutica, además de restaurar la salud y proteger la vida.

Finalmente, es importante resaltar que la praxis de enfermería está ligada a la vocación, la axiología y los preceptos humanistas; y que es necesario la auto reflexión e interreflexividad para mejorar diariamente el 
accionar enfermero en nuestros establecimientos de salud.

\section{Referencias}

Agrazal, J. (2016). Ser enfermera: ciencia y vocación para una atención humanizada. Panamá: La estrella de Panamá. Recuperado de: http://laestrella.com.pa/opinion/columnistas/enfermera-cienciavocacion-para-atencion-humanizada/23939510

Camejo, R. (2017). Análisis Prospectivo de la Gerencia de Enfermería desde las Voces de los Estudiantes. Revista Scientific, 2(5), 242-261. Recuperado de:

https://doi.org/10.29394/scientific.issn.2542-2987.2017.2.5.13.242-261 Constitución de la República Bolivariana de Venezuela (1999). Gaceta Oficial de la República Bolivariana de Venezuela, № 5.908. (Extraordinario), febrero 9, 2009. Caracas, Venezuela: La Asamblea Nacional de la República Bolivariana de Venezuela. Recuperado de:

http://www.asambleanacional.gob.ve/documentos archivos/constitucio n-nacional-7.pdf

Guerrero, R., Meneses, M., \& De La Cruz, M. (2015a,b). Cuidado humanizado de enfermería según la teoría de Jean Watson. Revista Enfermería Herediana, 9(2), 133-142. Recuperado de:

https://doi.org/10.20453/renh.v9i2.3017

Guillaumet, O., García, I., Casacuberta, M., \& Serra, M. (2005). Teoría del cuidado humano. Un café con Watson. Metas de enfermería, ISSN: 1138-7262, 8(2), págs. 28-32. Recuperado de:

https://dialnet.unirioja.es/servlet/articulo?codigo $=1112382$

Kohlberg, L. (1997a,b). La educación moral según Lawrence Kohlberg. Barcelona, España: Gedisa.

Ley Orgánica de Educación (2009). Gaceta Oficial de la República Bolivariana de Venezuela, $\mathbf{N}^{\circ}$ 5.929, (Extraordinario), agosto 15. 
Carcas, Venezuela: La Asamblea Nacional de la República Bolivariana de Venezuela. Recuperada de:

http://www.ucv.ve/fileadmin/user upload/asesoria juridica/LOE.pdf

López, R., \& Torres, M. (2009a,b). Praxis en enfermería: Implicaciones. Revista Enfermería Universitaria ENEO-UNAM, 6(4), 1-6. Recuperado de: http://www.revistas.unam.mx/index.php/reu/article/view/29904

Masi, A. (2008). El concepto de praxis en paulo freire. Consejo Latinoamericano de Ciencias Sociales, Buenos Aires: Red de Bibliotecas Virtuales de Ciencias Sociales de América Latina y el Caribe de la Red CLACSO. ISBN: 978-987-1183-81-4. Recuperado de:

http://bibliotecavirtual.clacso.org.ar/ar/libros/campus/freire/09Masi.pdf

Núñez, K. (2017). Filosofía del cuidado humanizado de Jean Watson. Boletín de Enfermería. San José, Costa Rica: Colegio de Enfermeras de Costa Rica. Recuperado de: http://boletin.enfermeria.cr/jean-watsonexpuso-su-filosofia-del-cuidado-humanizado-a-la-enfermeriacostarricense/

Reluz, F., \& Cajachahua, L. (2011a,b). Humanismo Constructivista - Jean Piaget / Lawrence Kohlberg y Programas Tutoriales. Revista Consensus 16(1), 1-14. Recuperado de:

http://www.unife.edu.pe/pub/consensus/consensus16/luiscajachahua.p df 


\section{Rubén Eliut Hernández Ortega \\ e-mail: rubenho1977@gmail.com}

Nacido en la Güiria estado Sucre, Venezuela.

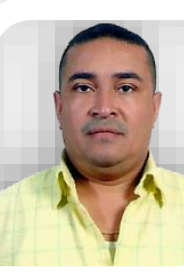
Licenciado en Enfermería (Universidad Nacional Experimental Rómulo Gallegos) UNERG, Magister Scientiarum en Educación Superior (Caribbean International University) CIU, Estudiante del programa de Doctorado en Ciencias de la Educación (Universidad Latinoamericana y del Caribe) ULAC, Coordinador del PNF de Enfermería del Distrito Capital (2012-2016), Docente del PNF de Enfermería (Universidad Politécnica Territorial de Los Altos Mirandinos "Cecilio Acosta"), Coordinador Docente de la Dirección Nacional de Enfermería del Ministerio del Poder Popular para la Salud (MPPS), Coordinador de Post Grados de Enfermería de la Universidad Ciencias de la Salud. Supervisor de Enfermería en el Hospital Psiquiátrico Dr. Jesús Mata de Gregorio del Instituto Venezolano de los Seguros Sociales (IVSS).

El contenido de este manuscrito se difunde bajo una Licencia de Creative Commons Reconocimiento- 